\title{
Polynomial scaling enhancement in the ground-state preparation of Ising spin models via counterdiabatic driving
}

\author{
Andreas Hartmann $\bullet^{1,},{ }^{*}$ Glen Bigan Mbeng, ${ }^{1, \dagger}$ and Wolfgang Lechner ${ }^{1,2, \dagger}$ \\ ${ }^{1}$ Institut für Theoretische Physik, Universität Innsbruck, Technikerstraße 21a, 6020 Innsbruck, Austria \\ ${ }^{2}$ Parity Quantum Computing GmbH, Rennweg 1, 6020 Innsbruck, Austria
}

(Received 12 October 2021; accepted 15 December 2021; published 18 February 2022)

\begin{abstract}
The preparation of ground states of spin systems is a fundamental operation in quantum computing and serves as the basis of adiabatic quantum computing. This form of quantum computation is subject to the adiabatic theorem which in turn poses a fundamental speed limit. We show that by employing diabatic transitions via counterdiabatic driving, a less strict requirement on adiabaticity applies. We demonstrate a scaling advantage from local and multispin counterdiabatic driving in the ground-state fidelity compared to their adiabatic counterpart, for different Ising spin models.
\end{abstract}

DOI: 10.1103/PhysRevA.105.022614

\section{INTRODUCTION}

Recent advances in the field of quantum technology [1-5] have paved the way for quantum supremacy in the so-called noisy intermediate-scale quantum (NISQ) computing regime [6,7]. Adiabatic quantum computation is a computational method for NISQ devices with the aim to solve combinatorial optimization problems [8]. Adiabatic quantum computation is subject to the adiabatic theorem $[9,10]$, which relates the minimal energy gap between the ground state and the first-excited eigenstate and the time to perform an adiabatic evolution. This gap decreases exponentially with the system size at a first-order quantum phase transition (QPT) and polynomially at a second-order QPT $[11,12]$. Thus, the time to solution increases exponentially with the size of the system. Recent approaches to bypass this fundamental bottleneck have been dubbed diabatic quantum annealing (QA) [13]. This metaheuristic aims at solving combinatorial optimization problems in finite time, i.e., it is not restricted to adiabatic driving, thus allowing diabatic transitions between the system eigenstates during the annealing process $[14,15]$. The inherent issue with the latter approach, however, is that the success probability to reach the ground state of the system at the end of the QA process decreases considerably, thus leading to a potentially wrong solution to the combinatorial optimization problem of interest.

A particular form of diabatic quantum annealing is based on counterdiabatic (CD) driving [16-19]. Here the underlying idea is to suppress unwanted transitions during such

\footnotetext{
*andreas.hartmann@uibk.ac.at

$\dagger$ glen.mbeng@uibk.ac.at

ॠolfgang.lechner@uibk.ac.at
}

Published by the American Physical Society under the terms of the Creative Commons Attribution 4.0 International license. Further distribution of this work must maintain attribution to the author(s) and the published article's title, journal citation, and DOI. finite-time annealing processes by an additional term in the Hamiltonian [20-23]. In general, in order to obtain the exact form of the latter, which suppresses all transitions between any eigenstates and thus to always find the classical final ground state at the end of the sweep, even for very short durations, one needs to have a priori knowledge of the system eigenstates at all times during the annealing process, which introduces a severe computational and experimental hindrance for the implementation in near-term quantum devices. To overcome this, a variational approach to find approximate CD Hamiltonians has been introduced recently [24-26] and successfully implemented in different experimental setups $[27,28]$. Although approximate CD Hamiltonians are numerically and experimentally much easier to implement, the question of how their performance in finding the ground state scales with the system size is still open.

In this work we present a polynomial scaling advantage in the ground-state fidelity using approximate $\mathrm{CD}$ driving compared to its QA counterpart for Ising spin models with nearest-neighbor and all-to-all connected interactions. For the former, we simulate large system sizes up to 100 spins using numerical methods based on matrix product states (MPSs). We numerically demonstrate that the exponential complexity of QA reduces to another exponential complexity for approximate $\mathrm{CD}$ driving, with both local one-spin and nonlocal two-spin terms, yet with a considerably smaller coefficient. We further show for the Ising spin model with all-to-all connectivity that a similar behavior can be reached, depending on the strength of the interactions between the spins. We further provide a discussion on the nature of the additionally applied CD Hamiltonians and their cost of implementation. The numerical results reveal a strong polynomial enhancement of the applied approximate CD method, thus serving as an exciting starting point for implementation in near-term quantum annealing devices.

This work is structured as follows. In Sec. II we introduce the methods of approximate CD driving and MPSs. We present our numerical results in Sec. III. We summarize our 
results and give an outlook on future research in Sec. IV. Further details are provided in the Appendix.

\section{METHODS}

Quantum annealing is a metaheuristic to solve combinatorial optimization problems. In its most general form, it can be written as a time-dependent Hamiltonian of the form

$$
\begin{aligned}
\mathcal{H}_{0}(t) & =[1-\lambda(t)] \mathcal{H}_{\mathrm{d}}+\lambda(t) \mathcal{H}_{\mathrm{p}}, \\
\mathcal{H}_{\mathrm{d}} & =-\sum_{j=1}^{N} \gamma_{j} \sigma_{j}^{x},
\end{aligned}
$$

where $\mathcal{H}_{\mathrm{d}}$ is the driver Hamiltonian with site-dependent $\sigma^{x}$ and transverse magnetic-field strength $\gamma_{i}$, and $N$ is the total number of spins in the system. On the other hand, $\mathcal{H}_{\mathrm{p}}$ is the problem Hamiltonian in the $z$ direction that encodes our optimization problem of interest. Throughout this work, we use the driving function

$$
\lambda(t)=\sin ^{2}\left[\frac{\pi}{2} \sin ^{2}\left(\frac{\pi t}{2 \tau}\right)\right],
$$

which fulfills $\lambda(t=0)=0$ and $\lambda(t=\tau)=1$ as well as $\dot{\lambda}(t=$ $0)=\dot{\lambda}(t=\tau)=\ddot{\lambda}(t=0)=\ddot{\lambda}(t=\tau)$, with $\tau$ the sweep duration in order to obtain a smooth function. The time to find the lowest-energy eigenstate, i.e., the ground state, of the problem Hamiltonian $\mathcal{H}_{\mathrm{p}}$ to solve the optimization problem is subject to the adiabatic theorem $[9,10]$. While for long, i.e., adiabatic, sweep durations the annealing schedule $\mathcal{H}_{0}(t)$ [Eq. (1)] of the system remains in the true ground state, the same is not true for shorter times when the success probability drops significantly. This dilemma between employing sufficiently short sweep durations while at the same time reaching a high ground-state fidelity constitutes a severe issue within this field.

To bypass this bottleneck, we additionally employ a counterdiabatic Hamiltonian to speed up QA. In particular, we drive the quantum system with the total Hamiltonian

$$
\mathcal{H}(t)=\mathcal{H}_{0}(t)+\mathcal{H}_{\mathrm{CD}}(t),
$$

where $\mathcal{H}_{0}(t)$ [Eq. (1)] is the original Hamiltonian and $\mathcal{H}_{\mathrm{CD}}(t)$ the additional CD Hamiltonian that aims to suppress transitions between the system's eigenstates and thus to follow the instantaneous ground state of the system. This time-dependent CD Hamiltonian reads

$$
\mathcal{H}_{\mathrm{CD}}(t)=\dot{\lambda}(t) \mathcal{A}_{\lambda}(t),
$$

where $\mathcal{A}_{\lambda}(t)$ is the exact adiabatic gauge potential (AGP) [24-26] and $\dot{\lambda}(t)$ is the derivative of the sweep function $\lambda(t)$ of Eq. (2). In this work, we employ approximate AGPs $\mathcal{A}_{\lambda}^{\prime}$ that are much easier to build numerically and experimentally yet do not fully prevent the system from inducing transitions between the eigenstates during the procedure. The corresponding approximate CD Hamiltonians entail $p$-spin terms with an odd number of $\sigma^{y}$ terms and $p \leqslant N$. As an example, for $p=1$ we only introduce site-dependent one-spin $\sigma_{j}^{y}$ terms, whereas for $p=2$, we additionally employ two-spin $\sigma_{j}^{y} \sigma_{k}^{x}$ and $\sigma_{j}^{y} \sigma_{k}^{z}$ terms. Employing all combinations of up to $N$-spin terms, i.e., $p=N$, provides us with the exact AGP. In order to obtain the optimal form of these approximate AGPs, we apply an Ansatz and minimize the operator distance between the exact AGP and the parameters of the Ansatz (see the Appendix for more details).

This method can be applied to various problem Hamiltonians $\mathcal{H}_{\mathrm{p}}$. Here we consider two different Ising spin models with (i) nearest-neighbor and (ii) all-to-all connected interactions in the problem Hamiltonian $\mathcal{H}_{\mathrm{p}}$, respectively.

The problem Hamiltonian of the Ising spin model with nearest-neighbor interactions and $N$ total spins reads

$$
\mathcal{H}_{\mathrm{p}}^{\mathrm{NN}}=-\sum_{j=1}^{N} b_{j} \sigma_{j}^{z}-\sum_{j=1}^{N-1} J_{j} \sigma_{j}^{z} \sigma_{j+1}^{z},
$$

where $\sigma_{j}^{z}$ is the $z$-Pauli matrix at site $j, b_{j}$ is the applied site-dependent longitudinal magnetic field strength, and $J_{j}$ is the interaction strength for which we impose open boundaries, i.e., $\sigma^{N+1}=0$. This Ising spin model is described by a onedimensional short-range Hamiltonian and thus we can employ numerical methods based on MPSs [29-33]. In particular, we use a time-evolving block decimation (TEBD) $[34,35]$ with a second-order Trotter expansion of the Hamiltonian to simulate the dynamics in the MPSs.

As a second Ising spin model, we consider nonlocal interactions with all-to-all connectivity. The corresponding problem Hamiltonian of interest reads

$$
\mathcal{H}_{\mathrm{p}}^{\text {all }}=-\sum_{j=1}^{N} b_{j} \sigma_{j}^{z}-\sum_{j=1}^{N} \sum_{k<j} J_{j k} \sigma_{j}^{z} \sigma_{k}^{z},
$$

where $b_{j}$ is again the longitudinal magnetic-field strength at site $j$ and $J_{j k}$ is the interaction strength between spins at sites $j$ and $k$. The uniform case with $J_{i j}=1$ and $b_{j}=0$ was considered in Refs. [36,37]. However, here we consider the more general case where the $b_{j}$ are chosen randomly. Due to the long-range interactions $J_{j k}$, methods based on MPSs cannot be applied efficiently, which restricts our numerical simulations to small system sizes. For both models, we consider 1-local $(p=1)$ and 2-local potential $(p=2)$ approximate AGPs. We provide the explicit expressions of the two approximate AGPs for the nearest-neighbor model, in Sec. 1 of the Appendix, and for the all-to-all model, in Sec. 2 of the Appendix.

\section{NUMERICAL RESULTS}

In this section we highlight the performance of finite-time quantum annealing, written in the form of the original Hamiltonian $\mathcal{H}_{0}(t)$ [Eq. (1)] and various counterdiabatic protocols $\mathcal{H}(t)=\mathcal{H}_{0}(t)+\mathcal{H}_{\mathrm{CD}}(t)$ with both local one-spin $(p=1)$ and nonlocal two-spin $(p=2)$ CD Hamiltonians as in Eq. (4), for different system sizes. To this end, we compute for each protocol the success probability, i.e., the final ground-state fidelity

$$
F(\tau)=\left|\left\langle\psi(\tau) \mid \phi_{0}(\tau)\right\rangle\right|^{2},
$$

as the absolute value of the overlap of the state reached $|\psi(\tau)\rangle$ and $\left|\phi_{0}(\tau)\right\rangle$, the actual ground state at the end of the sweep with duration $\tau$. For the Ising spin chain, we use imaginary TEBD to compute a MPS representation of the ground state $\left|\phi_{0}(\tau)\right\rangle$. 


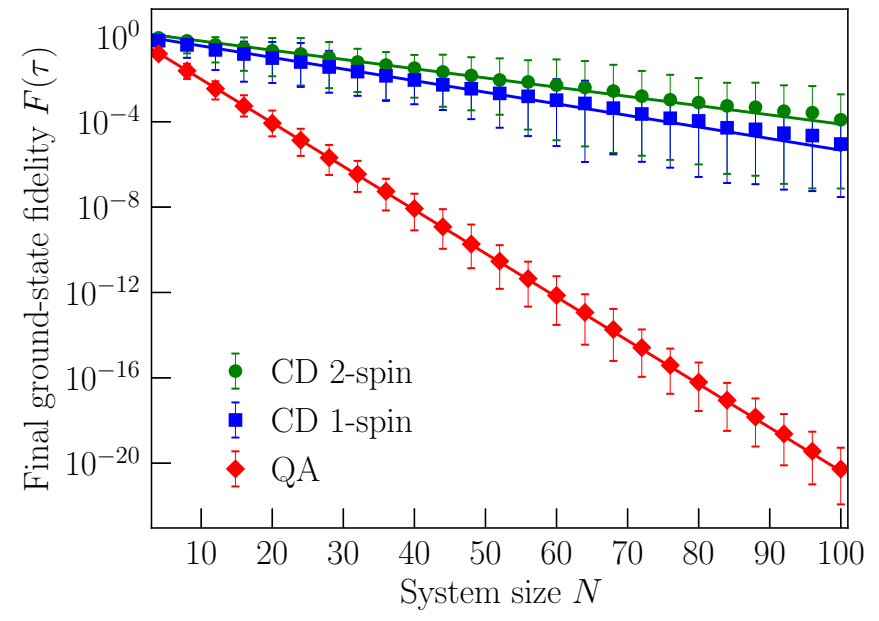

FIG. 1. Final ground-state fidelity over system size for the nearest-neighbor model. The mean final ground-state fidelity $F(\tau)$ [Eq. (7)] is plotted over the system size $N$ for the nearest-neighbor Ising spin model with (i) the original Hamiltonian $\mathcal{H}_{0}^{\mathrm{NN}}(t)$ [Eq. (5), red diamonds], (ii) the full Hamiltonian with local one-spin $\mathrm{CD} \mathcal{H}_{\mathrm{CD}}^{(1)}$ [Eq. (4), blue squares], and (iii) the nonlocal two-spin CD protocol $\mathcal{H}_{\mathrm{CD}}^{(2)}$ [Eq. (4), green circles], taken over an ensemble of 50 randomly chosen instances of longitudinal magnetic fields $b_{j}$, drawn from a Gaussian distribution with mean zero and standard deviation one, for sweep duration $\tau=10$. The corresponding solid lines depict the fitted exponential curves for each driving protocol with values $a \approx 0.47$ for QA, $b \approx 0.13$ for one-spin $\mathrm{CD}$ driving, and $c \approx 0.1$ for two-spin CD driving, respectively.

\section{A. Nearest-neighbor model}

First, we will start with the nearest-neighbor model with the original Hamiltonian $\mathcal{H}_{0}^{\mathrm{NN}}(t)$ [Eq. (5)]. The sweep duration is set to $\tau=10$ and the interaction strength to $J_{j}=0.5$, thus describing a ferromagnet. To simulate the dynamics of the system we use a TEBD algorithm with a Trotter time step of $\Delta t=0.05$ and a maximum bond dimension $\chi=100$. The bond dimension $\chi$ controls the maximum entanglement allowed in the MPS and should be sufficiently large to accurately describe the annealing dynamics of the system [32]. To ensure the validity of our numerical computation, for all the system sizes considered $(N \leqslant 100)$, we verified that the results do not depend on either $\chi$ or $\Delta t$.

Figure 1 depicts the final ground-state fidelity $F(\tau)$ reached [Eq. (7)] for (i) the original Hamiltonian $\mathcal{H}_{0}^{\mathrm{NN}}(t)$ [Eq. (5), red diamonds], (ii) the full Hamiltonian with the local one-spin CD protocol $\mathcal{H}_{\mathrm{CD}}^{(1)}(t)$ [Eq. (4), blue squares], and (iii) the nonlocal two-spin CD protocol $\mathcal{H}_{\mathrm{CD}}^{(2)}(t)$ [Eq. (4), green circles] for an ensemble of 50 randomly chosen longitudinal magnetic fields $b_{j}$, drawn from a Gaussian distribution with mean zero and standard deviation one, for various system sizes $N$ up to 100 spins. For this nonadiabatic sweep duration of $\tau=10$, the mean of the final ground-state fidelities for QA with system size $N=100$ drops to $F_{\mathrm{QA}}(\tau) \approx 10^{-20}$, whereas for $\mathrm{CD}$ driving it remains at $F_{\mathrm{CD}}^{(1)} \approx 10^{-4}$ for local one-spin and $F_{\mathrm{CD}}^{(2)} \approx 10^{-3}$ for nonlocal two-spin $\mathrm{CD}$ driving. The numerical results reveal a significant enhancement in the ground-state fidelity for the local one-spin and nonlocal two-spin CD-driven protocols compared to their quantum annealing counterpart. In particular, the results show a scaling advantage in the sense that the original exponential size complexity $F_{0}(\tau) \propto \exp (-a N)$ reduces to another exponential complexity yet with a smaller coefficient, i.e., $F_{\mathrm{CD}}^{(1)}(\tau) \propto$ $\exp (-b N)$ with $b<a$ for local one-spin CD driving and $F_{\mathrm{CD}}^{(2)}(\tau) \propto \exp (-c N)$ with $c<b<a$ for nonlocal two-spin $\mathrm{CD}$ driving. In particular, we have fitted these curves, i.e., the exponent $s$ of the function $F(N)=r \exp (-s N)$, and received the values $a \approx 0.47$ for $\mathrm{QA}, b \approx 0.13$ for one-spin $\mathrm{CD}$ driving, and $c \approx 0.1$ for two-spin $\mathrm{CD}$ driving, which are plotted as solid lines in Fig. 1. This scaling advantage of the final ground-state fidelity for local and nonlocal CD driving over quantum annealing persists for various coupling regimes $\left(J_{j}=0.1\right.$ for the weak-coupling regime, $J_{j}=0.5$ for the intermediate-coupling regime, and $J_{j}=1$ for the strongcoupling regime; see the Appendix).

\section{B. All-to-all connected model}

Let us now consider the Ising spin model with all-to-all connected interactions $J_{j k}$ and original Hamiltonian $\mathcal{H}_{0}^{\text {all }}(t)$ [Eq. (6)] with transverse magnetic-field strengths as well as the interactions strengths between each combination of sites $j$ and $k$ with $\gamma_{j}=J_{j k}=1$. The sweep duration is $\tau=1$. Figure 2(a) depicts the final ground-state fidelity $F(\tau)$ reached [Eq. (7)] for (i) the original Hamiltonian $\mathcal{H}_{0}^{\text {all }}(t)$ [Eq. (6), red diamonds] and the full Hamiltonians with (ii) the local one-spin CD protocol $\mathcal{H}_{\mathrm{CD}}^{(1)}$ [Eq. (4), blue squares] and (iii) the nonlocal two-spin CD protocol $\mathcal{H}_{\mathrm{CD}}^{(2)}$ [Eq. (4), green circles] for an ensemble of 50 randomly chosen longitudinal magnetic fields $b_{j}$, drawn from a Gaussian distribution with mean zero and standard deviation one, for various system sizes $N$ up to eight spins. The mean of the final ground-state fidelities for quantum annealing approximately scale as $F_{\mathrm{QA}}(\tau) \propto 1 / 2^{N}$. This is the parameter regime of the quench limit where the spins randomly flip from the $x$ into the $z$ direction, each pointing with probability $\frac{1}{2}$ into the right direction. Local CD driving provides a constant speedup compared to QA. On the other hand, the fidelities with nonlocal two-spin CD driving depict a polynomial scaling enhancement for increasing system sizes. For this model, the fitted exponents in the exponential curves read $a \approx 0.47 \approx b$ for $\mathrm{QA}$ and local one-spin $\mathrm{CD}$ driving, respectively, and $c \approx 0.1$ for nonlocal two-spin $\mathrm{CD}$ driving. Figure 2(b) depicts the quotient of the final ground-state fidelity reached for $\mathrm{CD}$ and traditional QA driving, i.e., $F(\tau) / F_{\mathrm{QA}}(\tau)$, where for convenience we provided a guide for the eye. In this panel, we see that a scaling advantage for nonlocal two-spin CD driving in the final ground-state fidelities reached can be reached towards their traditional QA counterpart.

The numerical results reveal a considerable enhancement in the ground-state fidelity, similar to systems with nearestneighbor interaction. Whereas for the latter the polynomial enhancement persists also for local one-spin CD driving, this feature ceases to exist for interactions with all-to-all connectivity due to its increased complexity and seems to diminish to a constant speedup only. However, for nonlocal two-spin $\mathrm{CD}$ driving the polynomial enhancement is observed as the approximate AGP also contains two-spin terms to account for the dominance of the all-to-all connected interactions. 

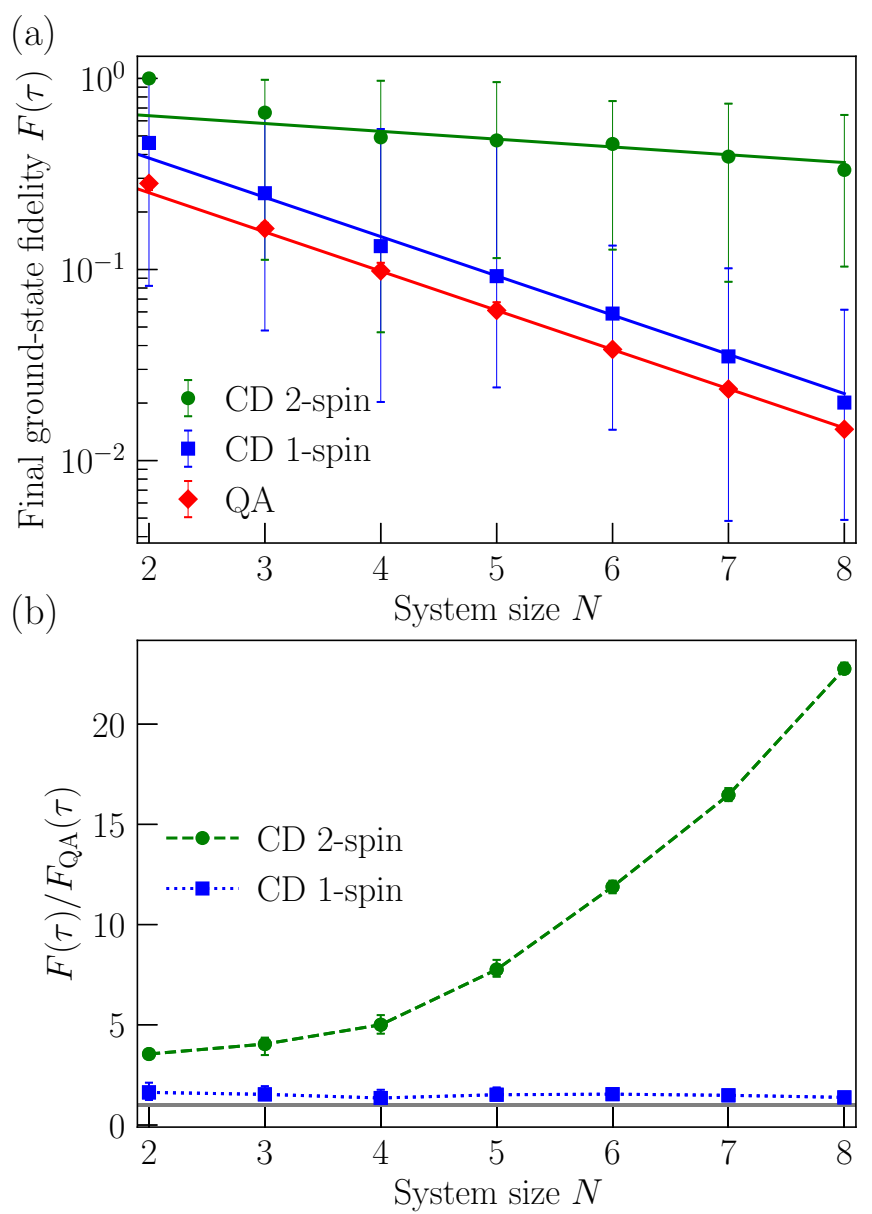

FIG. 2. Final ground-state fidelity over system size for the all-toall model. (a) Mean final ground-state fidelity $F(\tau)$ [Eq. (7)] over the system size $N$ for the all-to-all connected Ising spin model for (i) the original Hamiltonian $\mathcal{H}_{0}^{\text {all }}(t)$ [Eq. (6), red diamonds], (ii) the full Hamiltonian with the local one-spin CD protocol $\mathcal{H}_{\mathrm{CD}}^{(1)}$ [Eq. (4), blue squares], and (iii) the nonlocal two-spin CD protocol $\mathcal{H}_{\mathrm{CD}}^{(2)}$ [Eq. (4), green circles], taken over an ensemble of 50 randomly chosen instances of longitudinal magnetic fields $b_{j}$, drawn from a Gaussian distribution with mean zero and standard deviation one. The solid lines depict the fitted exponential curves for each driving protocol with values $a \approx 0.47$ for $\mathrm{QA}, b \approx 0.47$ for one-spin $\mathrm{CD}$ driving, and $c \approx 0.1$ for two-spin CD driving, respectively. (b) Quotient of the ground-state fidelity reached for the $\mathrm{CD}$ and QA protocols, i.e., $F(\tau) / F_{\mathrm{QA}}(\tau)$. The interaction strength between all combinations of sites is set to $J_{j k}=1$ and the sweep duration to $\tau=1$. The error bars denote the largest and lowest values, respectively. The dotted and dashed lines are merely guides for the eye without fitting.

\section{DISCUSSION AND OUTLOOK}

In this work we have numerically demonstrated a scaling advantage in the ground-state fidelity using approximate local and nonlocal counterdiabatic driving compared to their quantum annealing counterparts, for two different classes of Ising spin models. The enhancement is strongly polynomial, meaning that the original exponential size complexity remains exponential for approximate CD driving, yet with a smaller coefficient. Remarkably, this feature also holds true for local CD driving whose full Hamiltonian can, by rotation around the $z$ axis, be brought into the form of a transverse field Ising model, consisting of merely $\sigma^{x}$ and $\sigma^{z}$ terms, yet with unconventional driving of the system parameters. We expect the scaling advantage to increase with larger $p$-spin CD driving, potentially up to an ideal exponential speedup for $N$-spin CD driving, where we adapt the exact adiabatic gauge potential, thus always tracking the instantaneous ground state for all sweep durations. This feature comes with an emerging cost of implementing the multispin CD protocols numerically as well as experimentally. The algebraic or numerical determination of the optimal parameters of the corresponding $\mathrm{CD}$ Hamiltonians and AGPs suffers from a polynomial overhead for increasing $p$-spin $\mathrm{CD}$ driving. On the other hand, implementing nonlocal CD Hamiltonians experimentally is hard to accomplish for current quantum annealing devices. In the end, these numerical results reveal a trade-off between a further enhancement of the performance and the cost of implementation of the additional nonlocal AGPs. The existing polynomial scaling advantage for the case of local CD driving compared to traditional QA encourages the experimental implementation of the former and may serve as an excellent candidate for efficient devices by using further modifications of these methods (cf. Refs. [37,38]), which is an interesting topic for future consideration.

\section{ACKNOWLEDGMENTS}

We thank J. Wurtz for fruitful discussions. This work was supported by the Austrian Science Fund through a START grant under Project No. Y1067-N27 and the SFB BeyondC Project No. F7108-N38, the Hauser-Raspe Foundation, and the European Union's Horizon 2020 research and innovation program under Grant Agreement No. 817482. This material is based upon work supported by the Defense Advanced Research Projects Agency (DARPA) under Contract No. HR001120C0068. Any opinions, findings and conclusions or recommendations expressed in this material are those of the author(s) and do not necessarily reflect the views of DARPA.

\section{APPENDIX: APPROXIMATE COUNTERDIABATIC DRIVING}

In order to find the optimal form of the adiabatic gauge potential $\mathcal{A}_{\lambda}(t)$ for the additional counterdiabatic Hamiltonian $\mathcal{H}_{\mathrm{CD}}(t)$ from Eq. (4), we employ the method introduced in Refs. [24,25]. In this approach, we rely on an approximate AGP $\mathcal{A}_{\lambda}^{\prime}(t)$ that can be derived from a variational principle. To this end, we define the Hermitian operator of interest as

$$
G_{\lambda}\left(\mathcal{A}_{\lambda}^{\prime}\right)=\partial_{\lambda} \mathcal{H}_{0}+i\left[\mathcal{A}_{\lambda}^{\prime}, \mathcal{H}_{0}\right],
$$

where we have set $\hbar \equiv 1$. With this, we can further define an operator distance between the exact solution for the AGP $\mathcal{A}_{\lambda}$ and our Ansatz for the approximate solution $\mathcal{A}_{\lambda}^{\prime}$ as the Frobenius norm

$$
\mathcal{D}\left(\mathcal{A}_{\lambda}^{\prime}\right)=\operatorname{Tr}\left\{\left[G_{\lambda}\left(\mathcal{A}_{\lambda}^{\prime}\right)+\mathcal{M}_{\lambda}\right]^{2}\right\}=\operatorname{Tr}\left[G_{\lambda}^{2}\left(\mathcal{A}_{\lambda}^{\prime}\right)\right]-\operatorname{Tr}\left(\mathcal{M}_{\lambda}^{2}\right),
$$

where $\mathcal{M}_{\lambda}$ describes the generalized force. In consequence, we aim to minimize this operator distance to find the optimal form of the approximate $\mathrm{AGP} \mathcal{A}_{\lambda}^{\prime}$. As the second term of 
Eq. (A2) is independent of the parameters in the Ansatz for the approximate AGP, minimization of this operator distance is equivalent to minimizing the action

$$
\mathcal{S}\left(\mathcal{A}_{\lambda}^{\prime}\right)=\operatorname{Tr}\left[G_{\lambda}^{2}\left(\mathcal{A}_{\lambda}^{\prime}\right)\right]
$$

with respect to the parameters of the Ansatz for $\mathcal{A}_{\lambda}^{\prime}$, symbolically written as

$$
\frac{\delta S\left(\mathcal{A}_{\lambda}^{\prime}\right)}{\delta \mathcal{A}_{\lambda}^{\prime}}=0,
$$

where the symbol $\delta$ depicts the functional derivative.

In this work we rely on two kinds of approximate CD Hamiltonians $\mathcal{H}_{\mathrm{CD}}(t)$ : first, a local one where the Ansatz for the AGP contains single-spin terms, and second, nonlocal Ansätze where the corresponding AGP contains multispin operators.

Implementation cost. The implementation of these additional CD Hamiltonians comes at a price, which we now consider. To estimate this cost, various quantifiers have been developed recently $[39,40]$. In this work we employ the quantifier

$$
\left\langle\mathcal{H}_{\mathrm{CD}}\right\rangle=v_{t, N} \int_{0}^{\tau} \operatorname{Tr}\left[\mathcal{H}_{\mathrm{CD}}^{\dagger}(t) \mathcal{H}_{\mathrm{CD}}(t)\right] d t
$$

that determines the power that is needed to generate the additional magnetic fields due to the presence of these CD Hamiltonians. Here $v_{t, N}$ is a highly setup-dependent parameter that in general is hard to determine and which is set to one during the following additional numerical results.

\section{Nearest-neighbor model}

For the Ising spin model with nearest-neighbor interactions $J_{j}$, we employ the original Hamiltonian

$$
\begin{aligned}
\mathcal{H}_{0}^{\mathrm{NN}}(t)= & -[1-\lambda(t)] \sum_{j=1}^{N} \gamma_{j} \sigma_{j}^{x} \\
& -\lambda(t)\left[\sum_{j=1}^{N} b_{j} \sigma_{j}^{z}+\sum_{j=1}^{N-1} J_{j} \sigma_{j}^{z} \sigma_{j+1}^{z}\right],
\end{aligned}
$$

where the driving function $\lambda(t)$ is chosen according to Eq. (2), fulfilling the conditions $\lambda(t=0)=0$ and $\lambda(t=\tau)=1$, with $\tau$ the sweep duration, $\gamma_{j}$ and $b_{j}$ the site-dependent transverse field and longitudinal magnetic-field strengths, respectively, and $J_{j}$ the interaction strength between spins at sites $j$ and $j+$ 1 , for which we apply open boundary conditions, i.e., $\sigma_{N+1}^{z}=$ 0 .

For this model we choose two types of AGPs: (i) a local single-spin $\mathcal{A}_{\lambda}^{(1)}$ Ansatz and (ii) nonlocal two-spin $\mathcal{A}_{\lambda}^{(2)}$ Ansatz for the approximate AGPs, i.e.,

$$
\begin{gathered}
\mathcal{A}_{\lambda}^{(1)}=\sum_{j=1}^{N} \alpha_{j} \sigma_{j}^{y}, \\
\mathcal{A}_{\lambda}^{(2)}=\sum_{j=1}^{N} \alpha_{j} \sigma_{j}^{y}+\sum_{j=1}^{N-1} \beta_{j} \sigma_{j}^{y} \sigma_{j+1}^{x}+\gamma_{j} \sigma_{j}^{x} \sigma_{j+1}^{y} \\
+\delta_{j} \sigma_{j}^{y} \sigma_{j+1}^{z}+\epsilon_{j} \sigma_{j}^{z} \sigma_{j+1}^{y},
\end{gathered}
$$

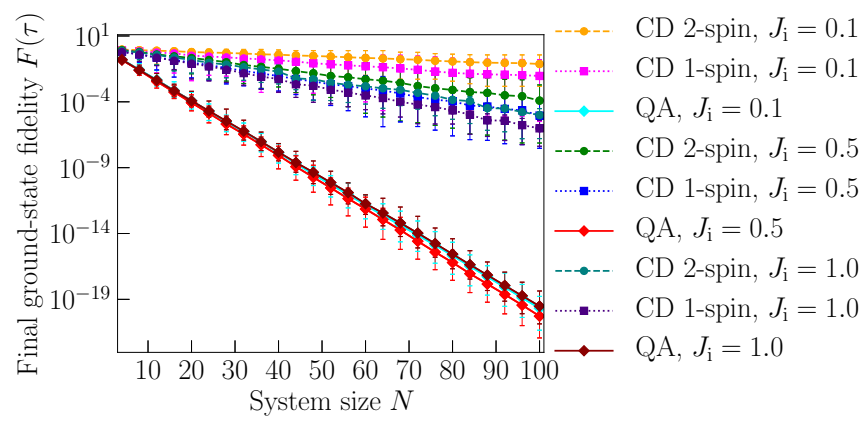

FIG. 3. Final ground-state fidelity over system size for the nearest-neighbor model. The mean final ground-state fidelity $F(\tau)$ [Eq. (7)] is plotted over the system size $N$ for the nearest-neighbor Ising spin model for (i) the original Hamiltonian $\mathcal{H}_{0}^{\mathrm{NN}}(t)$ [Eq. (5), solid lines with diamonds], (ii) the full Hamiltonian with the local one-spin $\mathrm{CD}$ protocol $\mathcal{H}_{\mathrm{CD}}^{(1)}$ [Eq. (4), dotted line with squares], and (iii) the nonlocal two-spin CD protocol $\mathcal{H}_{\mathrm{CD}}^{(2)}$ [Eq. (4), dashed line with circles], taken over an ensemble of 50 randomly chosen instances of longitudinal magnetic-field strengths $b_{j}$, drawn from a Gaussian distribution with mean zero and standard deviation one, for different interaction strengths $J_{j}$. The sweep duration is set to $\tau=10$ and the transverse magnetic-field strengths to $\gamma_{j}=1$. The error bars denote the highest and lowest values.

where we determine the site-dependent parameters $\alpha_{j}, \beta_{j}$, $\gamma_{j}, \delta_{j}$, and $\epsilon_{j}$ by minimizing the corresponding action (A3) numerically. For this model, the number of parameters to be determined is $N$ for the local Ansatz and $N+4(N-1)=$ $5 N-4$ for the nonlocal Ansatz.

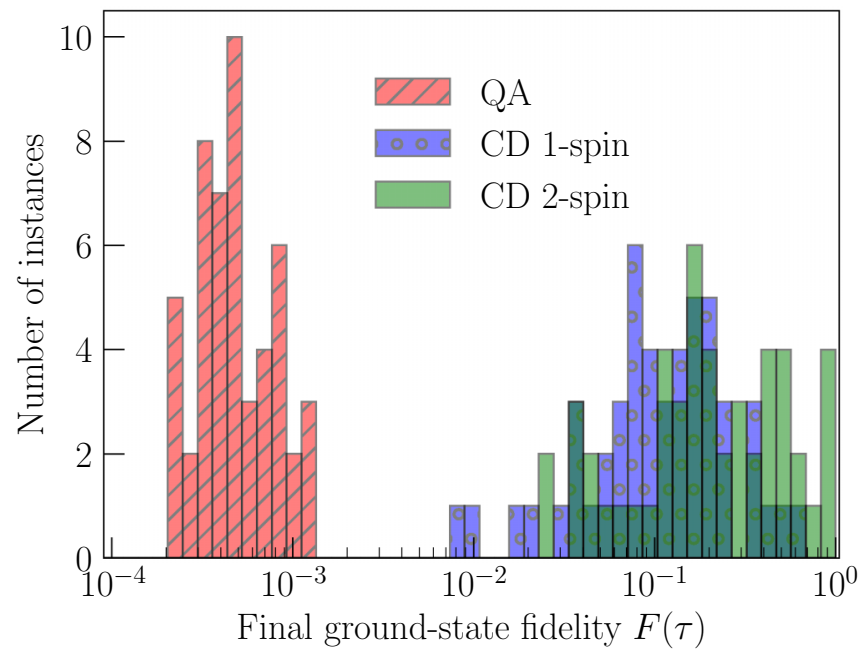

FIG. 4. Number of instances over ground-state fidelity. The histogram depicts the number of instances that attain a certain final ground-state fidelity $F(\tau)$ reached [Eq. (7)] for the nearest-neighbor Ising spin model for (i) the original Hamiltonian $\mathcal{H}_{0}^{\mathrm{NN}}(t)$ [Eq. (5), red stripes on the left], (ii) the full Hamiltonian with the local one-spin $\mathrm{CD}$ protocol $\mathcal{H}_{\mathrm{CD}}^{(1)}$ [Eq. (4), blue circles in the middle], and (iii) the nonlocal two-spin $\mathrm{CD}$ protocol $\mathcal{H}_{\mathrm{CD}}^{(2)}$ [Eq. (4), green solid bars at the right], taken over an ensemble of 50 randomly chosen instances of longitudinal magnetic fields $b_{j}$, drawn from a Gaussian distribution with mean zero and standard deviation one, for system size $N=16$ and other parameters as in Fig. 1. 


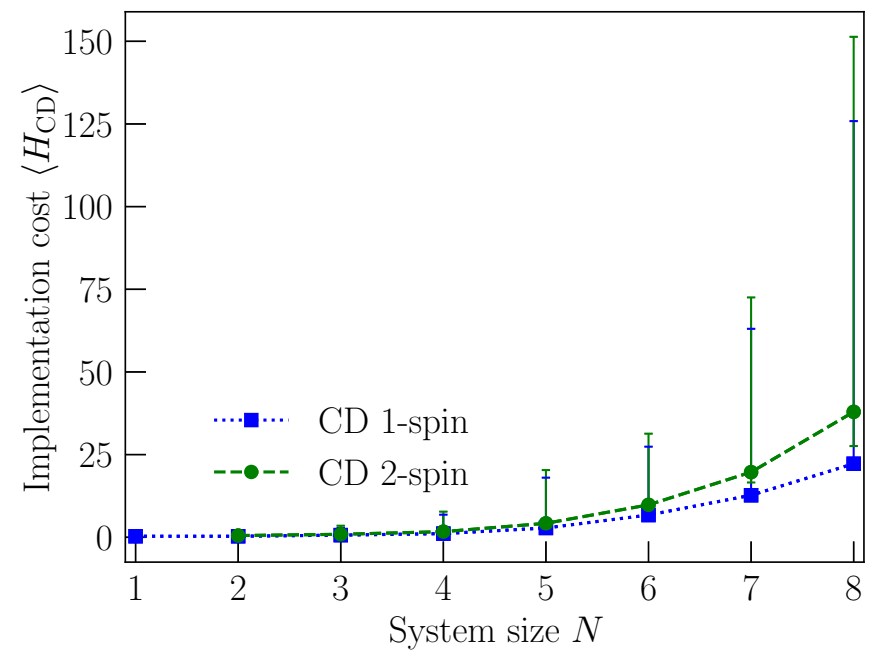

FIG. 5. Final ground-state fidelity. The mean final ground-state fidelity $F(\tau)$ [Eq. (7)] is plotted over the system size $N$ for the nearest-neighbor Ising spin model for (i) the full Hamiltonian with the local one-spin CD protocol $\mathcal{H}_{\mathrm{CD}}^{(1)}$ [Eq. (4), blue squares] and (ii) the nonlocal two-spin CD protocol $\mathcal{H}_{\mathrm{CD}}^{(2)}$ [Eq. (4), green circles], taken over an ensemble of 50 randomly chosen instances of longitudinal magnetic fields $b_{j}$, drawn from a Gaussian distribution with mean zero and standard deviation one. The sweep duration is set to $\tau=10$, the transverse magnetic-field strengths to $\gamma_{j}=1$, and the interactions strengths to $J=0.5$. The error bars denote the highest and lowest values.

Additional numerical results. Figure 3 depicts the final ground-state fidelity $F(\tau)$ reached [Eq. (7)] over the system size $N$ for the same parameters as in the main text (cf. Fig. 1 in Sec. III A) yet for different interaction strengths $J_{j}$, reaching from weakly $\left(J_{j}=0.1\right)$ via intermediate $\left(J_{j}=0.5\right)$ to strongly coupled spins $\left(J_{j}=1\right)$. The numerical results reveal that the polynomial scaling advantage in the final ground-state fidelity reached remains stable even for different interaction strengths, in particular, in the strong-coupling regime.

Furthermore, we are interested in the distribution of the final ground-state fidelities reached, which Fig. 4 depicts for system size $N=16$ and the same ensemble of longitudinal magnetic-field strengths $b_{j}$ chosen and other parameters as in Fig. 1. The numerical results show that the distribution of the ground-state fidelities $F(\tau)$ reached for the single instances can approximately be regarded as a Gaussian function for all three protocols, traditional quantum annealing (red bars), local one-spin CD driving (blue bars), and nonlocal two-spin $\mathrm{CD}$ driving (green bars). The fidelities reached for the latter two, however, are much higher compared to their traditional QA counterparts.

Figure 5 depicts the implementation cost $\left\langle\mathcal{H}_{\mathrm{CD}}\right\rangle$ [Eq. (A5)] for (i) local one-spin CD driving (blue squares) and (ii) nonlocal two-spin CD driving (green circles) for the same ensemble of instances and various system sizes $N$ as described in Sec. III A. We see that these implementation costs scale polynomially with increasing system size $N$ for both local and nonlocal CD driving. For the latter, the slope is larger as we have to take the additional $4(N-1)$ two-spin terms into

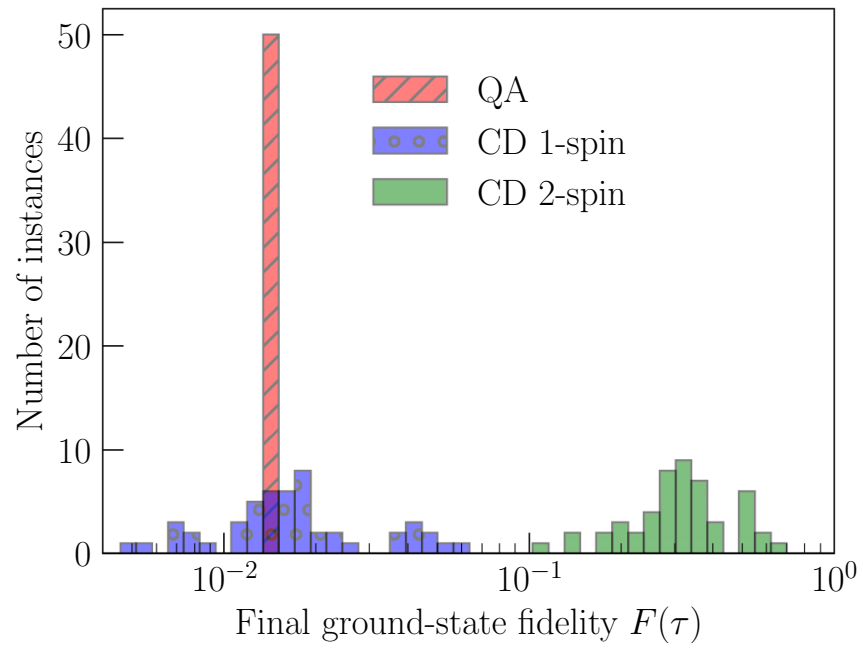

FIG. 6. Number of instances over ground-state fidelity. The number of instances that attain a certain final ground-state fidelity $F(\tau)$ [Eq. (7)] is plotted for the all-to-all connected Ising spin model for (i) the original Hamiltonian $\mathcal{H}_{0}^{\text {all }}(t)$ [Eq. (6), red stripes], (ii) the full Hamiltonian with local one-spin CD protocol $\mathcal{H}_{\mathrm{CD}}^{(1)}$ [Eq. (4), blue circles on the left], and (iii) the nonlocal two-spin $\mathrm{CD}$ protocol $\mathcal{H}_{\mathrm{CD}}^{(2)}$ [Eq. (4), green solid bars on the right], taken over an ensemble of 50 randomly chosen instances of longitudinal magnetic fields $b_{j}$, drawn from a Gaussian distribution with mean zero and standard deviation one, for system size $N=8$ and other parameters as in Fig. 2.

account compared to only $N$ one-spin terms from local CD driving.

\section{All-to-all connected model}

For the Ising spin model with all-to-all connectivity, we employ the original Hamiltonian

$$
\begin{aligned}
\mathcal{H}_{0}^{\text {all }}(t)= & -[1-\lambda(t)] \sum_{j=1}^{N} \gamma_{j} \sigma_{j}^{x} \\
& -\lambda(t)\left[\sum_{j=1}^{N} b_{j} \sigma_{j}^{z}+\sum_{j=1}^{N-1} \sum_{k>j} J_{j k} \sigma_{j}^{z} \sigma_{k}^{z}\right],
\end{aligned}
$$

where in analogy to the nearest-neighbor model we choose the driving function $\lambda(t)$ according to Eq. (2), $\gamma_{j}$ and $b_{j}$ describe the site-dependent transverse and longitudinal magnetic-field strengths, respectively, and $J_{j k}$ is the interaction strength between spins at sites $j$ and $k$.

In analogy to the nearest-neighbor model, we choose a local single-spin Ansatz $\mathcal{A}_{\lambda}^{(1)}$ and a nonlocal two-spin Ansatz $\mathcal{A}_{\lambda}^{(2)}$ for the approximate AGPs, i.e.,

$$
\begin{gathered}
\mathcal{A}_{\lambda}^{(1)}=\sum_{j=1}^{N} \alpha_{j} \sigma_{j}^{y}, \\
\mathcal{A}_{\lambda}^{(2)}=\sum_{j=1}^{N} \alpha_{j} \sigma_{j}^{y}+\sum_{j=1}^{N-1} \sum_{k>j} \beta_{j k} \sigma_{j}^{y} \sigma_{k}^{x}+\gamma_{j k} \sigma_{j}^{x} \sigma_{k}^{y} \\
+\delta_{j k} \sigma_{j}^{y} \sigma_{k}^{z}+\epsilon_{j k} \sigma_{j}^{z} \sigma_{k}^{y},
\end{gathered}
$$




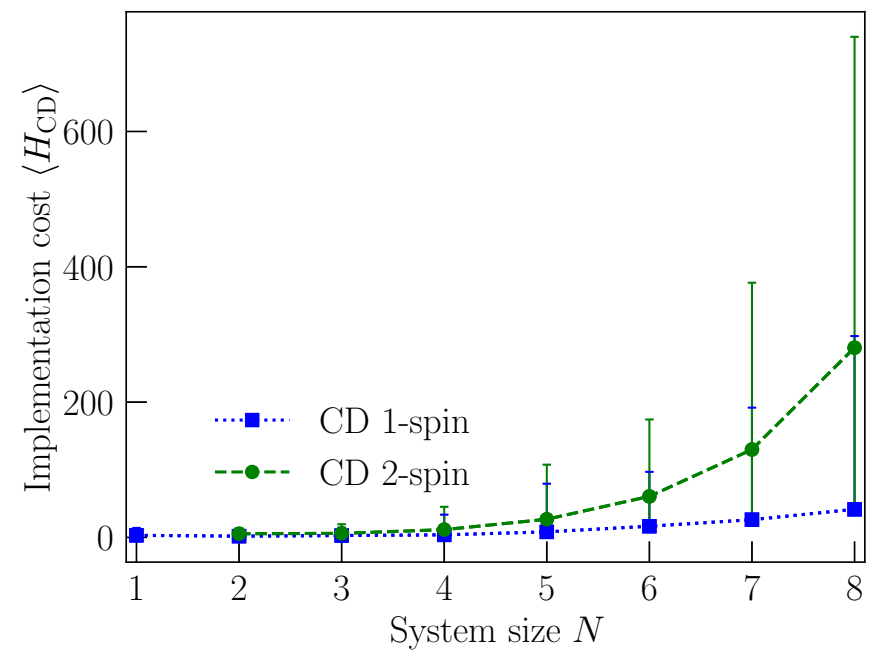

FIG. 7. Final ground-state fidelity. The mean final ground-state fidelity $F(\tau)$ [Eq. (7)] is plotted over the system size $N$ for the all-toall connected Ising spin model for (i) the full Hamiltonian with the local one-spin $\mathrm{CD}$ protocol $\mathcal{H}_{\mathrm{CD}}^{(1)}$ [Eq. (4), blue squares] and (ii) the nonlocal two-spin $\mathrm{CD}$ protocol $\mathcal{H}_{\mathrm{CD}}^{(2)}$ [Eq. (4), green circles], taken over an ensemble of 50 randomly chosen instances of longitudinal magnetic fields $b_{j}$, drawn from a Gaussian distribution with mean zero and standard deviation one. The sweep duration is set to $\tau=10$ and the transverse magnetic-field and interactions strengths are $\gamma_{j}=$ $J_{j k}=1$. The error bars denote the highest and lowest values.

where we determine the site- and interaction-dependent parameters $\alpha_{j}, \beta_{j k}, \gamma_{j k}, \delta_{j k}$, and $\epsilon_{j k}$ by minimizing the corresponding action (A3) numerically. For this model with all-to-all connected interactions and a nonlocal Ansatz of the corresponding AGP, the number of parameters to be determined is $N+4 N(N-1) / 2=4 N^{2}-N$ and thus it scales quadratically with the system size instead of linearly as for the local Ansatz and thus may constitute a numerical bottleneck for larger system sizes $N$.

Additional numerical results. Figure 6 depicts the distribution of the final ground-state fidelities $F(\tau)$ reached [Eq. (7)] for a system size $N=8$ and the same ensemble of chosen longitudinal magnetic-field strengths $b_{j}$ and other parameters as in Fig. 2. We see that the final ground-state fidelities reached for traditional quantum annealing (red bars) are all approximately equal, thus presenting the case of the quench limit where the fidelities scale as $F(\tau) \propto 1 / 2^{N}$. For local one-spin (blue) and nonlocal two-spin CD driving (green) the final fidelities reached are both Gaussian distributed. Whereas the fidelities for one-spin CD driving are only slightly larger than for the traditional quantum annealing counterpart while some instances perform even worse, all instances for the case of two-spin CD driving are considerably enhanced. Figure 7 depicts the implementation cost $\left\langle\mathcal{H}_{\mathrm{CD}}\right\rangle$ [Eq. (A5)] for the same ensembles and system sizes as described in Sec. III B, thus for (i) local CD driving (blue squares) and (ii) nonlocal $\mathrm{CD}$ driving (green circles). The numerical results show that these costs also increase polynomially with the system size, yet the slope of the nonlocal two-spin curve is greatly enlarged as, in contrast to the nearest-neighbor model, the number of parameters to be determined for the CD Hamiltonian now scales as $4 N^{2}-N$ and thus has a quadratic overhead compared to applying local terms only or the nonlocal terms in the nearest-neighbor model.
[1] I. Buluta and F. Nori, Quantum simulators, Science 326, 108 (2009).

[2] J. I. Cirac and P. Zoller, Goals and opportunities in quantum simulation, Nat. Phys. 8, 264 (2012).

[3] I. M. Georgescu, S. Ashhab, and F. Nori, Quantum simulation, Rev. Mod. Phys. 86, 153 (2014).

[4] M. Saffman, Quantum computing with atomic qubits and Rydberg interactions: Progress and challenges, J. Phys. B 49, 202001 (2016).

[5] J. Preskill, Quantum computing in the NISQ era and beyond, Quantum 2, 79 (2018).

[6] J. Preskill, Quantum computing and the entanglement frontier, arXiv:1203.5813.

[7] A. W. Harrow and A. Montanaro, Quantum computational supremacy, Nature (London) 549, 203 (2017).

[8] T. Albash and D. A. Lidar, Adiabatic quantum computation, Rev. Mod. Phys. 90, 015002 (2018).

[9] T. Kato, On the adiabatic theorem of quantum mechanics, J. Phys. Soc. Jpn. 5, 435 (1950).

[10] A. Messiah, Quantum Mechanics (North-Holland, Amsterdam, 1961), Vol. 2.

[11] J. Tsuda, Y. Yamanaka, and H. Nishimori, Energy gap at firstorder quantum phase transitions: An anomalous case, J. Phys. Soc. Jpn. 82, 114004 (2013).

[12] A. Mishra, T. Albash, and D. A. Lidar, Finite temperature quantum annealing solving exponentially small gap problem with non-monotonic success probability, Nat. Commun. 9, 2917 (2018).

[13] E. J. Crosson and D. A. Lidar, Prospects for quantum enhancement with diabatic quantum annealing, Nat. Rev. Phys. 3, 466 (2021).

[14] G. E. Santoro and E. Tosatti, Optimization using quantum mechanics: Quantum annealing through adiabatic evolution, J. Phys. A: Math. Theor. 39, R393 (2006).

[15] P. Hauke, H. G. Katzgraber, W. Lechner, H. Nishimori, and W. D. Oliver, Perspectives of quantum annealing: Methods and implementations, Rep. Prog. Phys. 83, 054401 (2020).

[16] E. Torrontegui, S. Ibáñez, S. Martínez-Garaot, M. Modugno, A. del Campo, D. Guéry-Odelin, A. Ruschhaupt, X. Chen, and J. G. Muga, in Advances in Atomic, Molecular, and Optical Physics, edited by E. Arimondo, P. R. Berman, and C. C. Lin (Academic, New York, 2013), Vol. 62, pp. 117-169.

[17] A. del Campo and K. Sengupta, Controlling quantum critical dynamics of isolated systems, Eur. Phys. J. Spec. Top. 224, 189 (2015).

[18] A. del Campo and K. Kim, Focus on shortcuts to adiabaticity, New J. Phys. 21, 050201 (2019).

[19] D. Guéry-Odelin, A. Ruschhaupt, A. Kiely, E. Torrontegui, S. Martínez-Garaot, and J. G. Muga, Shortcuts to adiabaticity: Concepts, methods, and applications, Rev. Mod. Phys. 91, 045001 (2019). 
[20] M. Demirplak and S. A. Rice, Adiabatic population transfer with control fields, J. Phys. Chem. A 107, 9937 (2003).

[21] M. Demirplak and S. A. Rice, Assisted adiabatic passage revisited, J. Phys. Chem. B 109, 6838 (2005).

[22] M. Demirplak and S. A. Rice, On the consistency, extremal, and global properties of counterdiabatic fields, J. Phys. Chem. 129, 154111 (2008).

[23] M. V. Berry, Transitionless quantum driving, J. Phys. A: Math. Theor. 42, 365303 (2009).

[24] D. Sels and A. Polkovnikov, Minimizing irreversible losses in quantum systems by local counterdiabatic driving, Proc. Natl. Acad. Sci. USA 114, E3909 (2017).

[25] M. Kolodrubetz, D. Sels, P. Mehta, and A. Polkovnikov, Geometry and non-adiabatic response in quantum and classical systems, Phys. Rep. 697, 1 (2017).

[26] P. W. Claeys, M. Pandey, D. Sels, and A. Polkovnikov, Floquet-Engineering Counterdiabatic Protocols in Quantum Many-Body Systems, Phys. Rev. Lett. 123, 090602 (2019).

[27] H. Zhou, Y. Ji, X. Nie, X. Yang, X. Chen, J. Bian, and X. Peng, Experimental Realization of Shortcuts to Adiabaticity in a Nonintegrable Spin Chain by Local Counterdiabatic Driving, Phys. Rev. Appl. 13, 044059 (2020).

[28] N. N. Hegade, K. Paul, Y. Ding, M. Sanz, F. AlbarránArriagada, E. Solano, and X. Chen, Shortcuts to Adiabaticity in Digitized Adiabatic Quantum Computing, Phys. Rev. Appl. 15, 024038 (2021).

[29] F. Verstraete and J. I. Cirac, Matrix product states represent ground states faithfully, Phys. Rev. B 73, 094423 (2006).

[30] D. Perez-Garcia, F. Verstraete, M. M. Wolf, and J. I. Cirac, Matrix product state representations, Quantum Inf. Comput. 7, 401 (2007).
[31] F. Verstraete, V. Murg, and J. Cirac, Matrix product states, projected entangled pair states, and variational renormalization group methods for quantum spin systems, Adv. Phys. 57, 143 (2008).

[32] U. Schollwöck, The density-matrix renormalization group in the age of matrix product states, Ann. Phys. (NY) 326, 96 (2011).

[33] R. Orús, A practical introduction to tensor networks: Matrix product states and projected entangled pair states, Ann. Phys. (NY) 349, 117 (2014).

[34] G. Vidal, Efficient Simulation of One-Dimensional Quantum Many-Body Systems, Phys. Rev. Lett. 93, 040502 (2004).

[35] A. J. Daley, C. Kollath, U. Schollwöck, and G. Vidal, Timedependent density-matrix renormalization-group using adaptive effective Hilbert spaces, J. Stat. Mech. (2004) P04005.

[36] G. Passarelli, V. Cataudella, R. Fazio, and P. Lucignano, Counterdiabatic driving in the quantum annealing of the $p$-spin model: A variational approach, Phys. Rev. Research 2, 013283 (2020).

[37] L. Prielinger, A. Hartmann, Y. Yamashiro, K. Nishimura, W. Lechner, and H. Nishimori, Two-parameter counter-diabatic driving in quantum annealing, Phys. Rev. Research 3, 013227 (2021).

[38] A. Hartmann and W. Lechner, Rapid counter-diabatic sweeps in lattice gauge adiabatic quantum computing, New J. Phys. 21, 043025 (2019).

[39] Y. Zheng, S. Campbell, G. De Chiara, and D. Poletti, Cost of counterdiabatic driving and work output, Phys. Rev. A 94, 042132 (2016).

[40] S. Campbell and S. Deffner, Trade-Off Between Speed and Cost in Shortcuts to Adiabaticity, Phys. Rev. Lett. 118, 100601 (2017). 